\title{
mTORC2 activation protects retinal ganglion cells via Akt signaling after autophagy induction in traumatic optic nerve injury
}

Yao-Tseng Wen ${ }^{1}$, Jia-Rong Zhang ${ }^{2}$, Kishan Kapupara ${ }^{1}$ and Rong-Kung Tsai ${ }^{1,3}$

\begin{abstract}
Traumatic optic neuropathy is an injury to the optic nerve that leads to vision loss. Autophagy is vital for cell survival and cell death in central nervous system injury, but the role of autophagy in traumatic optic nerve injury remains uncertain. Optic nerve crush is a robust model of traumatic optic nerve injury. p62 siRNA and rapamycin are autophagy inducers and have different neuroprotective effects in the central nervous system. In this study, p62 and rapamycin induced autophagy, but only p62 siRNA treatment provided a favorable protective effect in visual function and retinal ganglion cell (RGC) survival. Moreover, the number of macrophages at the optic nerve lesion site was lower in the p62siRNA-treated group than in the other groups. p62 siRNA induced more M2 macrophage polarization than rapamycin did. Rapamycin inhibited both mTORC1 and mTORC2 activation, whereas p62 siRNA inhibited only mTORC1 activation and maintained mTORC2 and Akt activation. Inhibition of mTORC2-induced Akt activation resulted in blood-optic nerve barrier disruption. Combined treatment with rapamycin and the mTORC2 activator SC79 improved RGC survival. Overall, our findings suggest that mTORC2 activation after autophagy induction is necessary for the neuroprotection of RGCS in traumatic optic nerve injury and may lead to new clinical applications.
\end{abstract}

\section{Introduction}

Traumatic optic neuropathy (TON) is damage to the optic nerve caused directly or indirectly by assault, head trauma, disasters, or road accidents. TON results in optic nerve edema, disrupting the pial vessels and consequently limiting the vascular supply. These events trigger an inflammatory response at the injury site, causing secondary damage to the optic nerve, which leads to axon degeneration and retinal ganglion cell (RGC) death ${ }^{1,2}$. Currently, there are no established treatment options for TON, but optic canal decompression and corticosteroids

\footnotetext{
Correspondence: Rong-Kung Tsai (rktsai@tzuchi.com.tw)

${ }^{1}$ Institute of Eye Research, Hualien Tzu Chi Hospital, Buddhist Tzu Chi Medical Foundation, Hualien, Taiwan

${ }^{2}$ Department of Ophthalmology, Hualien Tzu Chi Hospital, Buddhist Tzu Chi Medical Foundation, Hualien, Taiwan

Full list of author information is available at the end of the article.

These authors contributed equally: Yao-Tseng Wen, Jia-Rong Zhang
}

may provide limited support in secondary optic nerve injuries $^{3}$. Rodent optic nerve crush (ONC) is an excellent model that closely mimics human TON and exhibits similar pathophysiology, rendering it a useful tool for therapeutic and regenerative studies ${ }^{4-9}$.

Several studies have reported a crucial role of autophagy after optic nerve injury ${ }^{10-12}$. Within hours after optic nerve injury, there is a rapid increase in autophagic vesicles at the optic nerve lesion, which spread back to the RGC somata where LC3 levels are increased as early as $24 \mathrm{~h}$ after a lesion ${ }^{11}$. The transcriptional levels of autophagy-related genes such as Atg5 and Atg7 in RGC somata are increased between 3 and 10 days after a lesion $^{13}$. The highest activation of autophagy leads to RGC death in vivo ${ }^{11}$. In addition, a substantial increase in p62 in the optic nerve could be caused by insufficient autophagic flux or parallel upregulation of $\mathrm{p} 62^{14,15}$.

\section{(c) The Author(s) 2019}

(c) (i) Open Access This article is licensed under a Creative Commons Attribution 4.0 International License, which permits use, sharing, adaptation, distribution and reproduction c. in any medium or format, as long as you give appropriate credit to the original author(s) and the source, provide a link to the Creative Commons license, and indicate if changes were made. The images or other third party material in this article are included in the article's Creative Commons license, unless indicated otherwise in a credit line to the material. If material is not included in the article's Creative Commons license and your intended use is not permitted by statutory regulation or exceeds the permitted use, you will need to obtain permission directly from the copyright holder. To view a copy of this license, visit http://creativecommons.org/licenses/by/4.0/. 
According to our previous study, ethambutol induces impaired autophagic flux and RGC apoptosis in the rat retina ${ }^{16}$. In summary, autophagy is involved in RGC death after optic nerve injury. Thus, manipulation of autophagy in the injured optic nerve has therapeutic potential to preserve visual function.

mTORC1 activation results in autophagy inhibition ${ }^{17}$. Administering the autophagy inhibitor 3-methyladenine (3-MA) reduced axonal degeneration in the ONC model $^{12}$. Morgan-Warren et al. demonstrated that mTOR induction could improve RGC survival after $\mathrm{ONC}^{9}$. By contrast, induction of autophagy by rapamycin did not protect retinal neurons in an ischemia/reperfusion model ${ }^{18}$. Daily intraperitoneal administration of rapamycin before optic nerve axotomy was protective for RGCs ${ }^{14}$. Autophagy activation has a cytoprotective role in RGCs after traumatic optic nerve injury ${ }^{19}$ and in TNF-induced optic nerve degeneration ${ }^{20}$. Thus, the actual functions and consequences of autophagy in RGCs after optic nerve injury remain controversial.

In the present study, we selected two autophagy inducers, rapamycin and p62 siRNA, to explore the role of autophagy in the ONC model. Rapamycin can inhibit mTOR expression and activate autophagy in various cells $^{21}$. In addition, rapamycin directly inhibits mTORC1 activation; only chronic administration of rapamycin can inhibit mTORC2 but not in every cell line or tissue ${ }^{22}$. However, the neuroprotective effect of rapamycin remains uncertain in many optic nerve injury models. Sequestosome 1 (SQSTM1), also named P62 protein, is a ubiquitin-binding scaffold protein that colocalizes with aggregates of ubiquitinated protein; the protein is degraded by autophagic flux. P62 accumulates when autophagy is inhibited, and decreased levels can be observed when autophagy is induced ${ }^{23}$. p62 siRNA can facilitate autophagic flux to improve autophagy by reducing the accumulation of P62 aggregation ${ }^{24}$. Furthermore, p62 siRNA specifically inhibits mTORC1 assembly through a p62-raptor interaction to suppress mTORC1 activation ${ }^{25}$. Although p62 siRNA has not been applied clinically, several studies have revealed that p62 inhibition provides neuroprotective effects in optic nerve injury ${ }^{20,24,26}$. The present study investigated the role of autophagy by observing rapamycin-mediated and p62-mediated mTOR activation in a rat model of ONC.

\section{Methods}

\section{Animals}

Male Wistar rats (BioLASCO Co., Taiwan) weighing $150-180 \mathrm{~g}$ were used in this study. All animal care and surgical procedures were executed in accordance with the Association for Research in Vision and Ophthalmology Statement for the Use of Animals in Ophthalmic and Vision Research. The Institutional Animal Care and Use
Committee at Tzu Chi Medical Center approved all the animal experiments. All operations were performed with the animals under anesthesia, which was achieved by intramuscular administration of a ketamine $(100 \mathrm{mg} / \mathrm{kg}$ body weight) and xylazine (10 mg/kg body weight; Sigma, St. Louis, MO, USA) cocktail. The rats had free access to food and water in a controlled environment at a temperature of $23^{\circ} \mathrm{C}$ and $55 \%$ humidity with a 12-h light-dark cycle.

\section{ONC experiments}

ONC injury was induced as described in our previous reports $^{5-7}$. Briefly, the optic nerve was exposed, and a vascular clip (60-g microvascular clip, World Precision Instruments, FL, USA) was applied at a distance of $2 \mathrm{~mm}$ posterior to the eyeball for $30 \mathrm{~s}$. The surgery was performed carefully to avoid damage to the small vessels around the optic nerve. The rats were kept on electric heating pads at $37^{\circ} \mathrm{C}$ for recovery. The sham group of rats received optic nerve exposure without the crush operation.

\section{Study design}

For examining the neuroprotective effects of autophagy activators, ONC rats were treated with scrambled siRNA ( 50 pmol; $n=12$ ), p62 siRNA ( 50 pmol; $n=12$ ) or rapamycin ( $1 \mathrm{mM}$, Sigma-Aldrich, MO, USA; $n=12)$. To evaluate the therapeutic effect of mTORC2 activation in the rapamycin-treated group, mTORC2 activator SC79 (10 $\mu \mathrm{g}$, Sigma-Aldrich, MO, USA; $n=6)$ was injected into rapamycin-treated rats. To investigate blood-optic nerve barrier (BOB) protection by Akt activation, PI3K/Akt inhibitor LY294002 $(10 \mu \mathrm{g}$, EMD Millipore corp., MA, USA; $n=3$ ) was administered to p62-siRNA-treated rats. All siRNAs were purchased from GeneDireX (Las Vegas City, NV, USA). As mentioned, a single shot of drugs was administered through intravitreal injection immediately after ONC procedures. Twelve rats without ONC operation were allocated to the sham group. Rats were euthanized at the second week post-ONC for further analysis.

\section{Immunostaining for autophagy markers in the retina}

Retinal sections were first stained with LC3 and LAMP1 antibodies. The primary antibodies LC3 (1:100; Santa Cruz Biotechnology, Inc., USA) and LAMP1 (1:100, Abcam, Cambridge, MA, USA) were used. The secondary antibodies FITC (1:100; Kirkegaard and Perry, MD, USA) and Alexa 568 (1:200; Invitrogen) were used. Cell nuclei were counterstained with 4',6-diamidino-2-phenylindole (DAPI; 1:1000; Sigma, St. Louis, MO, USA).

\section{Quantitative reverse transcription-polymerase chain reaction}

Arg 1, CD206, and Fizz1 are markers of M2 macrophages $^{27}$. The Qiagen RNeasy Mini Kit was used to isolate 
tissue RNA from optic nerve lysates obtained through the sonication method. All RNA samples were reverse transcribed for $30 \mathrm{~min}$ at $42{ }^{\circ} \mathrm{C}$ with a High-Capacity cDNA Reverse Transcription Kit according to the standard protocol of the supplier (Applied Biosystems, Foster City, CA, USA). Quantitative reverse transcription-polymerase chain reaction (qRT-PCR) was performed using an $A B$ PRISM 7300 Sequence Detection System (Applied Biosystems, CA, USA) with the QuantiTect SYBR green qRTPCR kit (Qiagen, Hilden, Germany). The expression levels of each gene were normalized to the reference gene, CypA. The forward and reverse primers used in this study are provided in the supplementary file (Table S1).

\section{Flash visually evoked potentials (FVEPs)}

Flash visual evoked potentials (FVEPs) were recorded 2 weeks after ONC. A visual electrodiagnostic system (Espion, Diagnosys LLC, Gaithersburg, MA, USA) was used to measure FVEPs. The first positive wavelet was defined as the P1 wave, and the first negative wavelet was defined as the N1. The amplitudes of the P1-N2 wave were compared among the groups $(n=6$ rats in each group) to estimate visual function ${ }^{28}$.

\section{Retrograde labeling of RGCs with Fluoro-Gold}

The retrograde labeling of the RGCs was performed 1 week before the rats were euthanized. The detailed procedure has been described in our previous reports ${ }^{4-7,28}$. Briefly, $2 \mu \mathrm{L}$ of $5 \%$ Fluoro-Gold (Fluorochrome LLC, CO, USA) was injected into each superior colliculus. After euthanasia, RGCs were measured at a distance of 1 or $3 \mathrm{~mm}$ from the center of the optic nerve head to provide the central and midperipheral RGC densities, respectively. We calculated the numbers of RGCs in five randomly selected areas measuring $62,500 \mu \mathrm{m}^{2}$ each in the central and midperipheral regions of each retina, and we estimated their averages as the mean densities of RGCs in the central and midperipheral regions of each retina $(n=6$ in each group).

\section{In situ nick end labeling assay for apoptotic cell measurements}

Frozen sections were prepared with retinal samples cut at $1-2 \mathrm{~mm}$ sagittal from the optic nerve head to ensure the use of similar fields for comparison. ${ }^{29}$ An in situ nick end labeling (TUNEL) assay (DeadEndFluorometric TUNEL System, Promega Corporation, Madison, WI, USA) was used to detect apoptotic cells. TUNEL-positive cells in the RGC layer of each sample were counted in 10 high-powered fields (HPFs, $\times 400$ magnification).

\section{Immunostaining at the injury site of optic nerves}

The ED1 antibody reacted against extrinsic macrophages and intrinsic microglia ${ }^{28,30,31}$. Monoclonal antibodies against ED1 (1:100, Abcam, Cambridge, MA, USA) were used. Arg1 and CD206 are M2 macrophage markers $^{32}$. Polyclonal antibodies against Arg1 and CD206 (1:100, Abcam, Cambridge, MA, USA) were used to determine macrophage polarization. The samples were incubated in a primary antibody overnight at $4{ }^{\circ} \mathrm{C}$. The secondary antibody conjugated with fluorescein isothiocyanate (FITC and rhodamine, 1:100, Jackson ImmunoResearch Laboratories, West Grove, PA, USA) was incubated at room temperature for $1 \mathrm{~h}$. Counterstaining was performed using DAPI (1:1000, Sigma, St. Louis, MO, USA). For comparison, the ED1-positive cells were calculated in six HPFs ( $\times 400$ magnification) at the optic nerve lesion site.

\section{Western blotting}

Retinal samples were collected, homogenized, and then centrifuged at $15,000 \times g$ for $15 \mathrm{~min}$ at $4{ }^{\circ} \mathrm{C}$. Western blotting was used to measure the levels of LC3 (Santa Cruz Biotechnology, Inc., USA), LAMP1 (Abcam, Cambridge, MA, USA), p-mTOR S2448 (Cell Signaling, Beverly, MA, USA), p-S6K (Cell Signaling, Beverly, MA, USA), p-Rictor (Cell Signaling, Beverly, MA, USA), p-Akt S473 (Cell Signaling, Beverly, MA, USA), and p-GSK3b S9 (Cell Signaling, Beverly, MA, USA) in retinal samples. Retinal protein extracts were separated using a $4-12 \%$ NuPAGE Bis-Tris gel (Invitrogen, Carlsbad, CA, USA). The separated proteins were transferred onto polyvinylidene difluoride membranes and blocked with 5\% milk in Tris-buffered saline/Tween-20 containing $20 \mathrm{mM}$ Tris- $\mathrm{HCl}$ (pH 7.5), $0.5 \mathrm{M} \mathrm{NaCl}$, and $0.5 \%$ Tween-20. The membranes were then blotted with mouse anti-LC antibodies, mouse anti-LAMP1 antibody, mouse anti-p62 antibody, mouse anti-mTOR antibody, and goat antimouse immunoglobulin (Abcam, Cambridge, MA, USA). The blots were then developed with Enhanced Chemiluminescent Substrates (Perkin-Elmer Life Science, Boston, MA, USA), and the relative intensities of the bands were measured using an image analysis system (Amersham Biosciences Uppsala, Sweden).

\section{Transmission electron microscopy}

The ultrastructure of the capillary tight junction was observed as described in our previous report ${ }^{29}$. Sections measuring $80 \mathrm{~nm}$ in thickness were observed under a transmission electron microscope (Hitachi HighTechnologies Corporation, Japan). Five to six images were taken per sample at the desired magnification.

\section{Statistical analysis}

All measurements in this study were performed in a masked fashion. The Mann-Whitney $U$ test was used to evaluate the differences among groups in all experiments. Data are reported as the mean \pm standard deviation (SD), 
and statistical significance was confirmed if $p<0.05$. All statistical analyses were performed using commercial software (SPSS, Chicago, IL, USA).

\section{Results}

p62 knockdown and rapamycin induce autophagy in the retina after ONC

The autophagy induction efficacy of p62 siRNA and rapamycin was determined according to the mRNA levels of p62 and mTOR in retinas at 1 week after ONC. Both autophagy inducers reduced the mRNA expression level of p62 in the retina (Fig. 1a). Treatment with rapamycin reduced mTOR mRNA expression. However, this result was not observed in the p62-siRNA-treated group (Fig. 1a). In autophagy activation, three main steps must be triggered: the formation of an autophagosome; fusion of the autophagosome and a lysosome, also called an autolysosome; and degradation of the autolysosome. The autophagy marker protein LC3 is a readout for triggering the formation of an autophagosome. The autophagy marker protein LAMP1 is considered to be an indicator of the formation of an autolysosome. These two autophagy markers must be inspected when monitoring the condition of autophagy flux. Compared with treated with scrambled siRNA, treatment with either p62 siRNA or rapamycin induced higher levels of LC3 and LAMP1 (Fig. 1b). However, compared with treatment with scrambled siRNA, treatment with rapamycin only induced LC3 and LAMP1 expression in the RGC layer. Treatment with p62 siRNA induced LC3 and LAMP1 expression from the

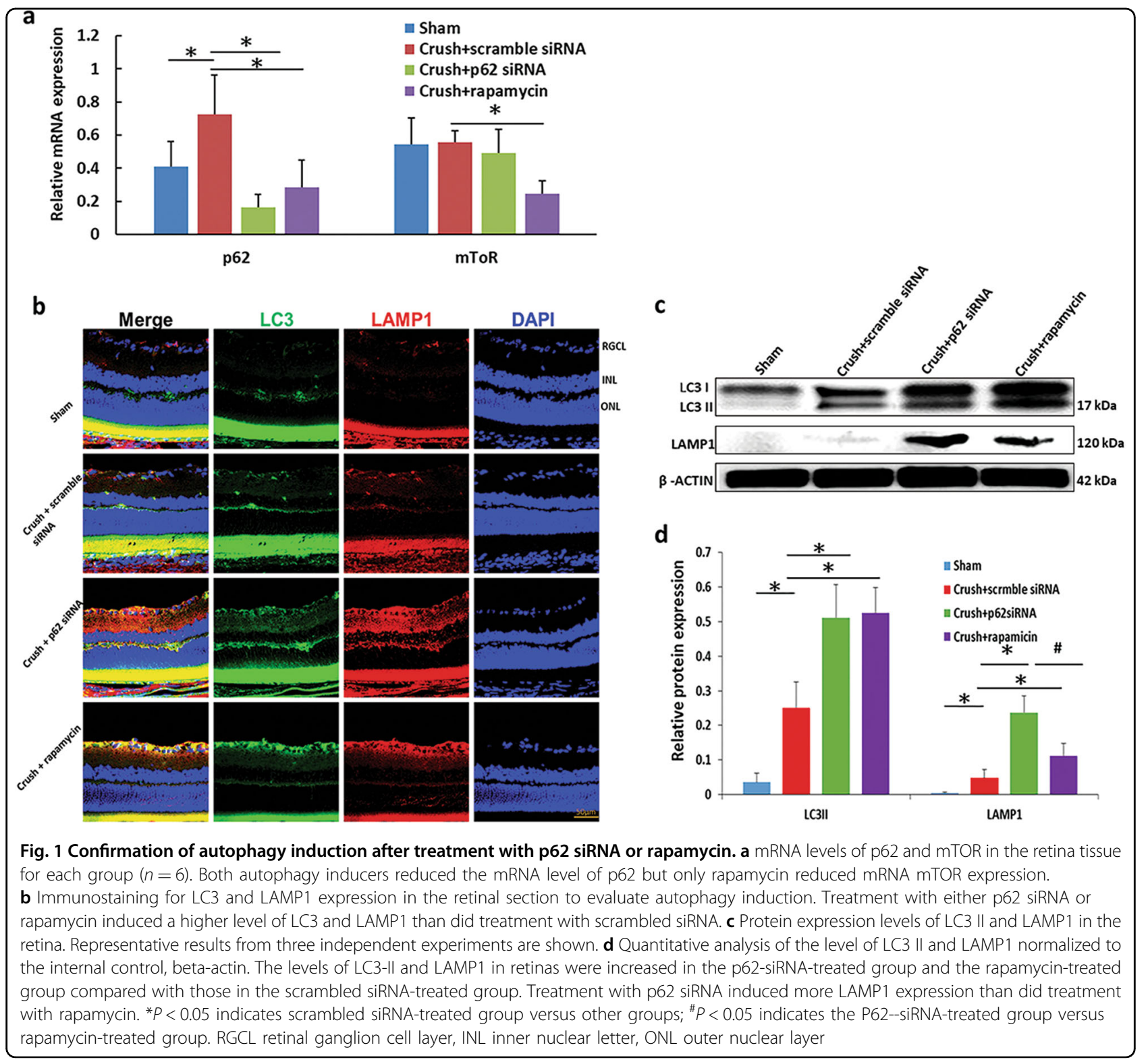




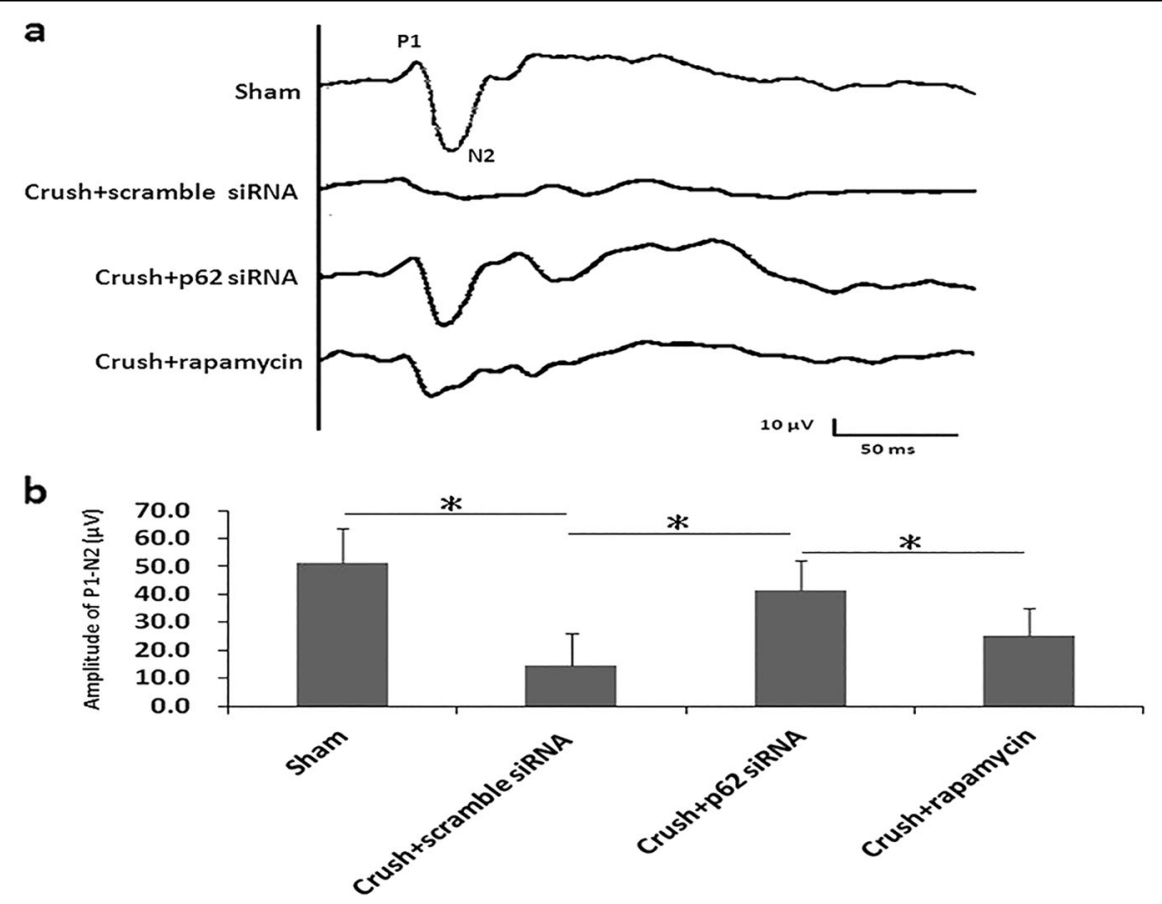

Fig. 2 Visual function preservation after treatment with p62 siRNA and rapamycin 2 weeks after ONC. a Representative FVEP tracings 2 weeks after ONC in the sham group, scrambled siRNA-treated group, p62 siRNA-treated group, and rapamycin-treated group. $\mathbf{b}$ Bar charts showing the P1N2 amplitude. Data are expressed as the mean \pm SD in each group $(n=6)$. The amplitudes of the P1-N2 waves in the p62 siRNA-treated group were significantly higher than those in the scrambled siRNA-treated group and rapamycin-treated group. ${ }^{*} P<0.05$

RGC layer to the outer nuclear layer in retinas. The expression of LC3 and LAMP1 demonstrated that autophagy was activated throughout the retina with p62 siRNA treatment compared with that observed after rapamycin treatment in ONC. To validate our findings, we performed Western blotting for LC-II and LAMP1. The levels of LC3-II in the retinas were increased by approximately twofold in the p62-siRNA-treated group and rapamycin-treated group compared with those in the scrambled siRNA-treated group $(p<0.05)$ (Fig. 1c). Treatment with p62 siRNA and rapamycin increased the LAMP1 expression level in retinas by approximately 5 fold and 2.5-fold, respectively $(p<0.05)$ (Fig. 1d). In addition, compared with treatment with rapamycin, treatment with p62 siRNA increased the LAMP1 expression level by approximately twofold $(p<0.05)$.

\section{Evaluation of visual function by FVEPs}

The FVEPs of the sham group and scrambled siRNAtreated, p62-siRNA-treated, and rapamycin-treated groups were recorded (Fig. 2a). The latency of the P1 wave was not significantly different among the groups in the FVEP tests. The amplitudes of the P1-N2 waves in the scrambled siRNA-treated, p62-siRNA-treated, and rapamycin-treated groups were $14.6 \pm 11.6,41.5 \pm 10.5$, and $25.2 \pm 9.8 \mu \mathrm{V}$, respectively. The P1-N2 amplitudes in the p62-siRNA-treated group were significantly higher than those in the scrambled-siRNA-treated group and rapamycin-treated group. However, the amplitudes of the P1-N2 waves showed no significant difference between the rapamycin-treated group and scrambled-siRNAtreated group (Fig. 2b).

\section{Only p62 siRNA treatment preserves the RGC survival rate and rescues $\mathrm{RGCs}$ from apoptosis}

Treatment with p62 siRNA preserved a higher density of RGCs in the central and midperipheral retina than did treatment with scrambled siRNA (Fig. 3a). The RGC densities in the central retinas of the sham group and scrambled siRNA-, p62-siRNA-treated, and rapamycintreated groups were $1576 \pm 185,536 \pm 196,1281 \pm 148$, and $673 \pm 144$ cells $/ \mathrm{mm}^{2}$, respectively. The RGC densities in the midperipheral retinas of the sham group and scrambled siRNA-, p62-siRNA-, and rapamycin-treated groups were $1017 \pm 173,301 \pm 230,832 \pm 148$, and $496 \pm$ 96 cells $/ \mathrm{mm}^{2}$, respectively (Fig. 3b). The numbers of RGCs in the central and midperipheral retina in the p62siRNA-treated group were 2.39-fold and 2.76-fold higher than those in the scrambled-siRNA-treated group, respectively. The numbers of RGCs in the central and midperipheral retinas in the p62-siRNA-treated group were 1.91-fold and 1.67-fold higher than those in the rapamycin-treated group, respectively. There was no significant difference in the numbers of RGCs between the 


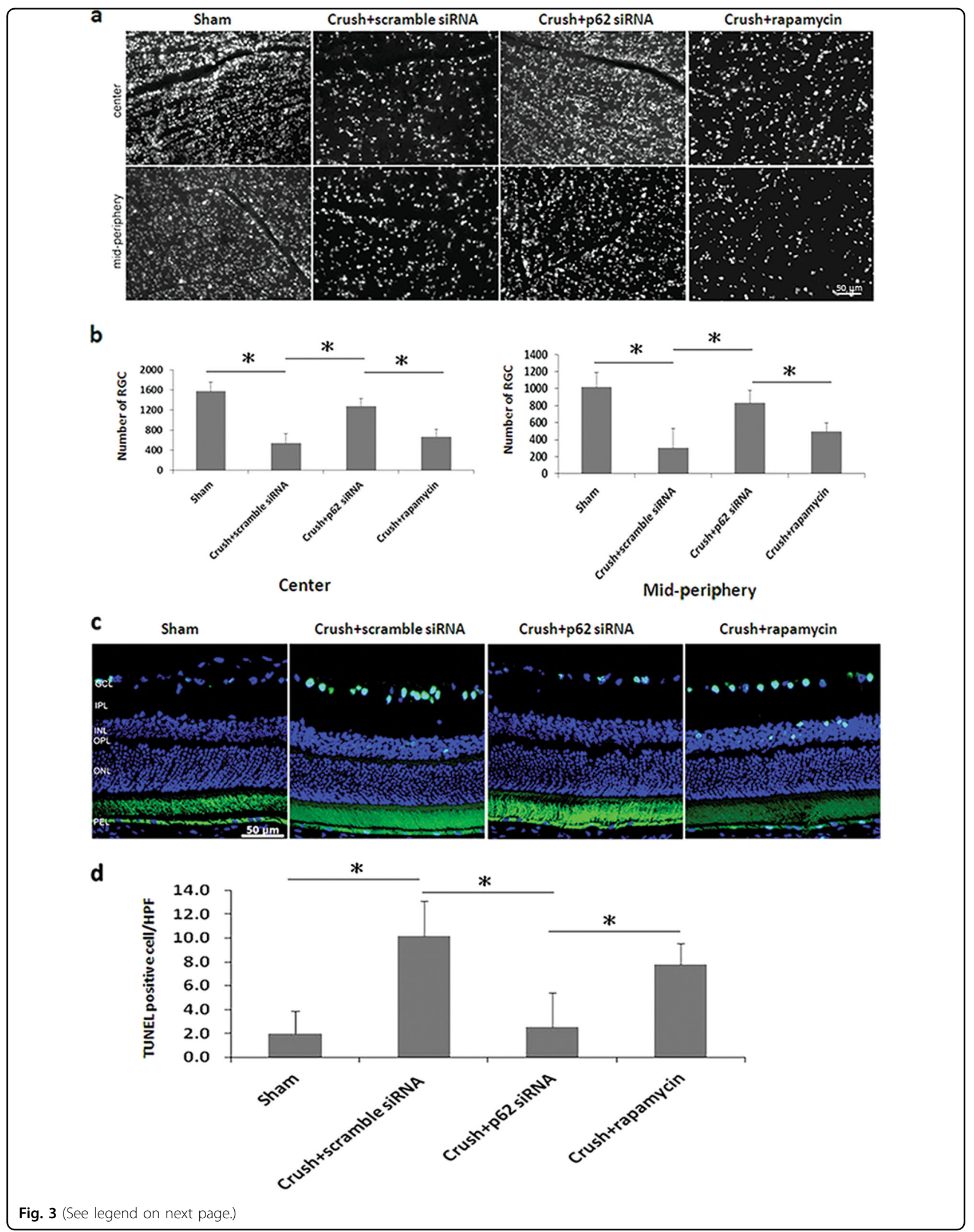




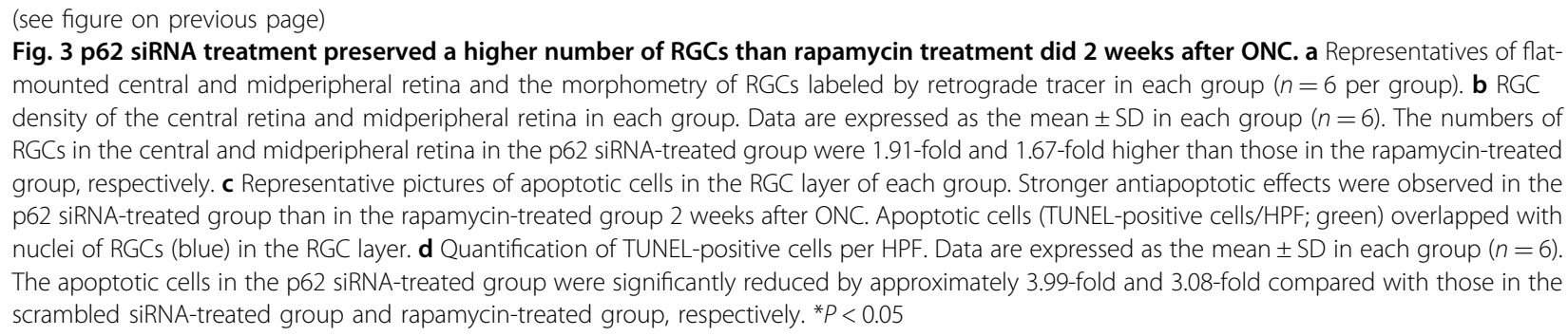

rapamycin-treated group and the scrambled-siRNAtreated group.

The numbers of TUNEL-positive cells in the RGC layer of the sham group and scrambled siRNA-treated, p62-siRNAtreated, and rapamycin-treated groups (Fig. 3c) were $2.0 \pm$ $1.9,10.2 \pm 2.9,2.5 \pm 2.8$, and $7.8 \pm 1.7$, respectively (Fig. $3 \mathrm{~d}$ ). The apoptotic cells in the p62-siRNA-treated group were significantly reduced by 3.99 -fold and 3.08-fold compared with those in the scrambled-siRNA-treated group and rapamycin-treated group, respectively (Fig. 3d). Similar to the RGC survival rate, there was no significant difference in apoptotic RGCs between the rapamycin-treated group and scrambled siRNA-treated group.

\section{Status of extrinsic macrophages and microglia after autophagy induction in the optic nerve}

The levels of extrinsic macrophages in the optic nerve indicate disruption of the $\mathrm{BOB}^{28,29}$, in addition to the anti-inflammatory efficacy of the neuroprotective agent. Moreover, microglia polarization toward the M2 state indicates anti-inflammatory activity ${ }^{28}$. The numbers of ED1-positive cells in the sham group and the scrambled siRNA-treated, p62-siRNA-treated, and rapamycintreated groups were $2.7 \pm 4.6,301.3 \pm 115.4,85.7 \pm 39.6$, and $277.0 \pm 154.6$, respectively (Fig. $4 \mathrm{a}, \mathrm{b}$ ). The macrophage recruitment in the p62-siRNA-treated group was reduced by 3.52-fold and 3.26-fold compared with that in the scrambled siRNA-treated group and rapamycintreated group, respectively. Furthermore, qRT-PCR results showed that Arg 1, CD206, and Fizz1 (markers for M2 microglia) were increased in the optic nerve after treatment with p62 siRNA compared with those observed after treatment with scrambled siRNA or rapamycin (Fig. 4c). Compared with treatment with scrambled siRNA, treatment with rapamycin also induced CD206 expression. In immunostaining analysis, the levels of CD206 and Arg1 were highly expressed in the optic nerve after immediate treatment with p62 siRNA compared with those observed after treatment with either scrambled siRNA or rapamycin (Fig. 4d). These results show that p62 knockdown halts macrophage infiltration and induces M2 polarization to a greater extent than rapamycin treatment does.

\section{mTORC2-mediated Akt activation is necessary for RGC survival after ONC}

mTOR activation was inhibited by treatment with either p62 siRNA or rapamycin after ONC (Fig. 5a, b). The activation of S6K (downstream of mTORC1) in the p62 siRNA-treated and rapamycin-treated groups was 2.81fold and 9.67-fold lower than that in the scrambled siRNA-treated group, respectively. The phosphorylation of Rictor, a key component of mTORC2, in the p62siRNA-treated group was 1.91-fold and 1.77-fold higher than that in the scrambled siRNA-treated and rapamycintreated groups, respectively. We observed no significant activation of Rictor in the rapamycin-treated group compared with that in the scrambled siRNA-treated group. The phosphorylation of Akt in the p62 siRNAtreated group was 3.14-fold and 34.83-fold higher than that in the scrambled siRNA-treated group and rapamycin-treated group, respectively. The phosphorylation of GSK3 $3-S 9$ in the p62 siRNA-treated group was 3.93-fold and 2.59-fold higher than that in the scrambled siRNA-treated group and rapamycin-treated group, respectively. These observations imply that p62 knockdown exerts neuroprotection via mTORC2-mediated Akt activation; however, rapamycin inhibited both mTORC1 and $\mathrm{mTORC} 2$, which resulted in reduced neuroprotective effects. Our previous findings showed BOB breakdown in the acute phase of retinal ischemia ${ }^{29}$. Hence, to evaluate the role of Akt activation in the acute phase of ONC, the capillary endothelial tight junction at the ONC site was examined. Compared with sham (Fig. S1a), one day after $\mathrm{ONC}$, the loss of the tight junction was observed in the capillaries of optic nerves (Fig. S1b). Treatment with p62 siRNA preserved the tight junction on day one after ONC (Fig. S1c). Combined treatment with p62 siRNA and Akt inhibitor did not preserve the tight junction one day after ONC (Fig. S1d), indicating that Akt activation is a critical process along with mTORC2 activation. The rapamycin-treated group was not examined for $\mathrm{BOB}$ integrity because treatment with rapamycin did not prevent macrophage infiltration in the optic nerve. Furthermore, compared with treatment with rapamycin alone, combined treatment with rapamycin and mTORC2 activator SC79 preserved the RGC survival rate in the central 


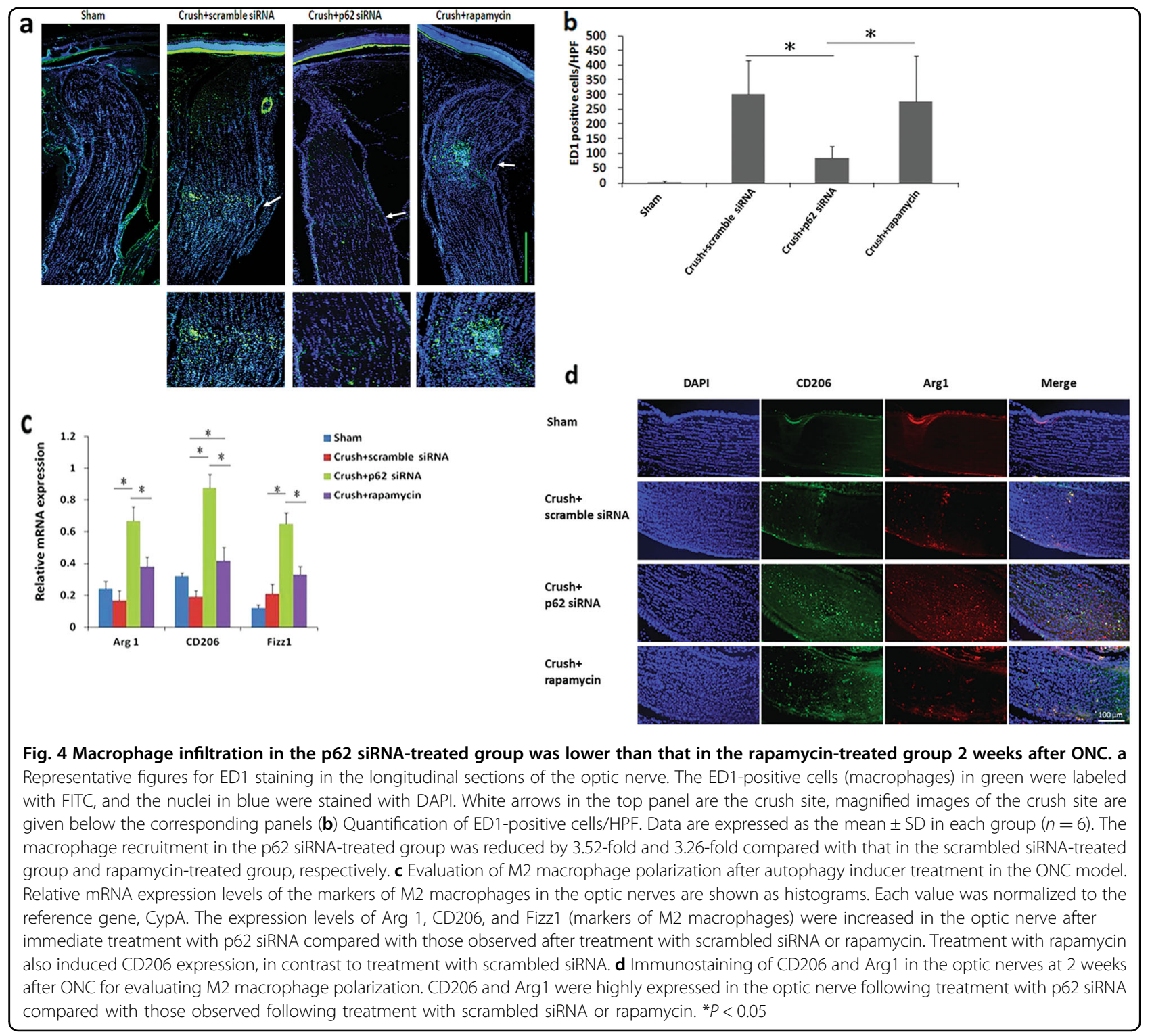

and midperipheral retins (Fig. S2a). The numbers of RGCs in the central and midperipheral retinas in the combined treatment group were 1.72-fold and 1.70-fold higher than those in the rapamycin-treated group, respectively (Fig. S2b).

\section{Discussion}

This study showed that intravitreal injections of an autophagy inducer (p62 siRNA) in an ONC model preserved more visual function than did treatment with another autophagy inducer (rapamycin) or scrambled siRNA. As expected, treatment with p62 siRNA rescued more RGCs and reduced more apoptotic RGCs in the retina than did treatment with rapamycin. Treatment with p62 siRNA had a more significant potency on autophagy induction than did treatment with rapamycin, as demonstrated by higher LAMP1 expression throughout the retina. Compared with rapamycin treatment, treatment with p62 siRNA significantly reduced macrophage infiltration into the optic nerve. Treatment with rapamycin reduced $\mathrm{mTORC} 1$ and $\mathrm{mTORC} 2$ activation. By contrast, p62 siRNA administration specifically inhibited mTORC1 activation and maintained mTORC2 activation after ONC. The activation of mTORC2 led to the phosphorylation of the Akt/GSK-3 $\beta$ signaling pathway. GSK$3 \beta$ phosphorylation via the PI3K/Akt pathway can reduce neuroinflammation and stabilize the blood-brain barrier $(\mathrm{BBB})^{33-35}$. Therefore, we conclude that treatment with p62 knockdown maintained mTORC2-mediated Akt activation to protect BOB integrity after ONC.

Both treatments induced M2 microglia/macrophage phenotypes, but treatment with p62 knockdown polarized 


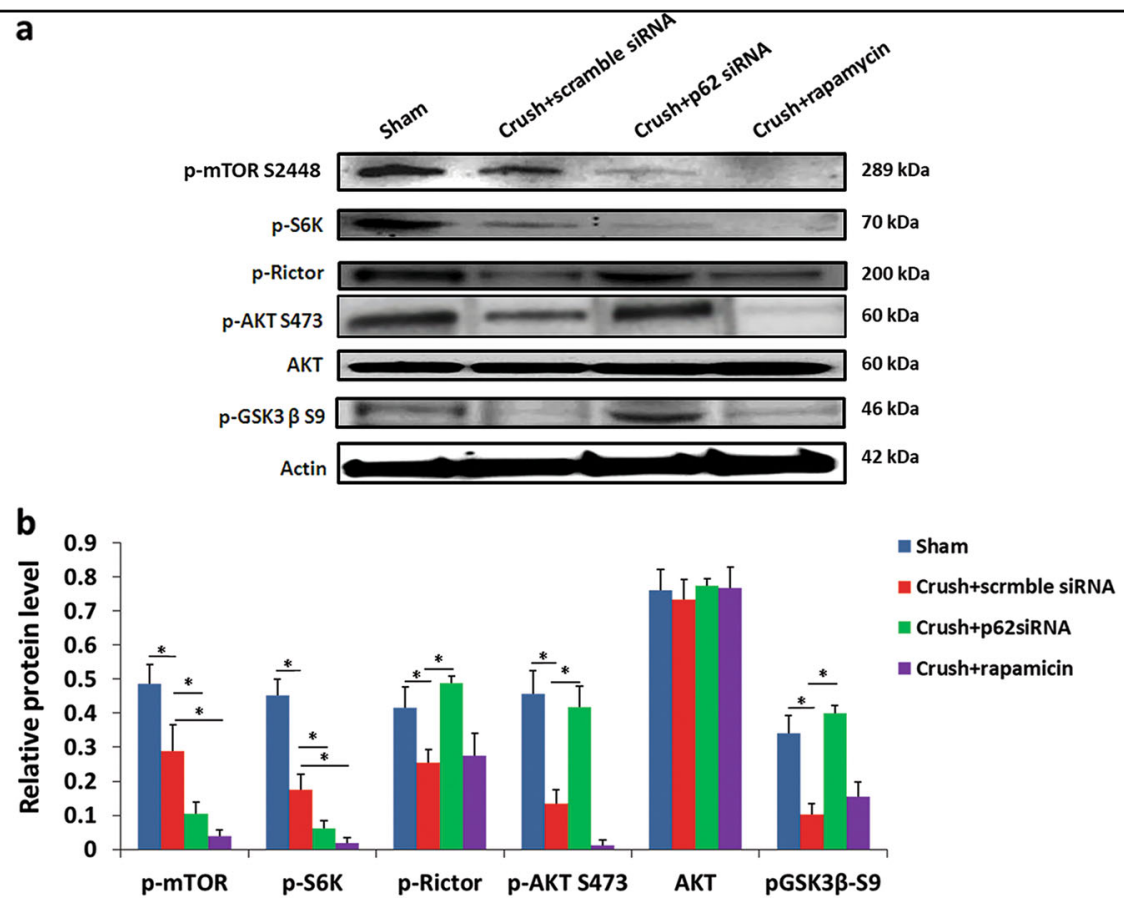

Fig. 5 Regulation of mTOR C1 and mTOR C2 activation in the retina after autophagy inducer treatment. a Western blotting of the expression levels of p-mTOR, p-S6K, p-Rictor, p-Akt S473, Akt, and p-GSK3 $\beta$ in each group. Representative results from three independent experiments are shown. b Quantitative analysis of the levels of p-mTOR, p-S6K, p-Rictor, p-Akt S473, Akt, and p-GSK3 $\beta$ normalized to the internal control, $\beta$-actin. ${ }^{*} P<0.05$

more M2 microglia/macrophage than did treatment with rapamycin. Toll-like receptor 2 signaling triggers M2 polarization and leads to NF- $\mathrm{kB}$ p65 degradation via a lysosome-dependent pathway ${ }^{36}$. This NF-kB p65containing aggresome-like structure is recognized by p62/SQSTM1 and degraded by selective autophagy ${ }^{36}$. Thus, autophagy induction may facilitate NF- $\mathrm{kB}$ p 65 degradation through p62 inhibition to improve M2 macrophage polarization. In sum, p62 knockdown reduced macrophage infiltration and neuroinflammation in the optic nerve to inhibit cytokine-induced apoptosis of RGCs through dual actions: mTORC2 activation stabilizing the $\mathrm{BOB}$ and M2 macrophage polarization.

The p62-raptor interaction elucidates why cells need p62 to activate mTORC1 in response to cell stimulation by amino acids $^{25}$. Thus, treatment with p62 knockdown selectively inhibits mTORC1 assembly. Our data also show that both autophagy inducers could induce autophagy and inhibit mTORC1. Although the functional association between autophagy and apoptosis remains unclear, a small connection shows that p62 levels are upregulated by autophagy deficiency or impairment ${ }^{10,23,37}$. Upregulation of p62 in the setting of autophagy impairment increases caspase-8-dependent apoptosis to improve its self-aggregation/activation and apoptosis ${ }^{38}$. Thus, treatment with p62 siRNA may inhibit p62 self-aggregation after optic nerve injury to prevent RGC apoptosis.
Research has reported that pretreatment of rapamycin by intraperitoneal injections can inhibit mTOR phosphorylation and preserve $\mathrm{RGCs}^{8,39}$. This slight preservation of RGCs is different from our observations. Another study on optic nerve axotomy also demonstrated that intraperitoneal injections of rapamycin preserved approximately $20 \%$ of RGCs on day 10 after optic nerve axotomy ${ }^{14}$. These studies have applied the same approach as intraperitoneal injections of rapamycin. Intraperitoneal injection of rapamycin before optic nerve injury induction may suppress the immune response by disrupting cytokine signaling ${ }^{40}$ to reduce optic nerve inflammationinduced damage. Morgan-Warren et al. reported that rapamycin reduced the RGC neuroprotective effects of siRTP801 in vitro 9 . Autophagy induction by intravitreal rapamycin administration did not provide RGC protection against ischemic stress ${ }^{18}$, which is consistent with our findings. This result suggests that intravitreal injection of rapamycin after optic nerve damage may not provide enough anti-inflammatory and immunosuppressive effects to protect RGCs.

Consistent with our findings, previous studies found that mTOR pathway modulation can assist axon regeneration after optic nerve injury through genetic deletion of PTEN $^{41}$ and delivery of fibroblast-derived exosomes ${ }^{42}$. Our strategy is slightly different from that in previous studies because we used autophagy activators to modulate 


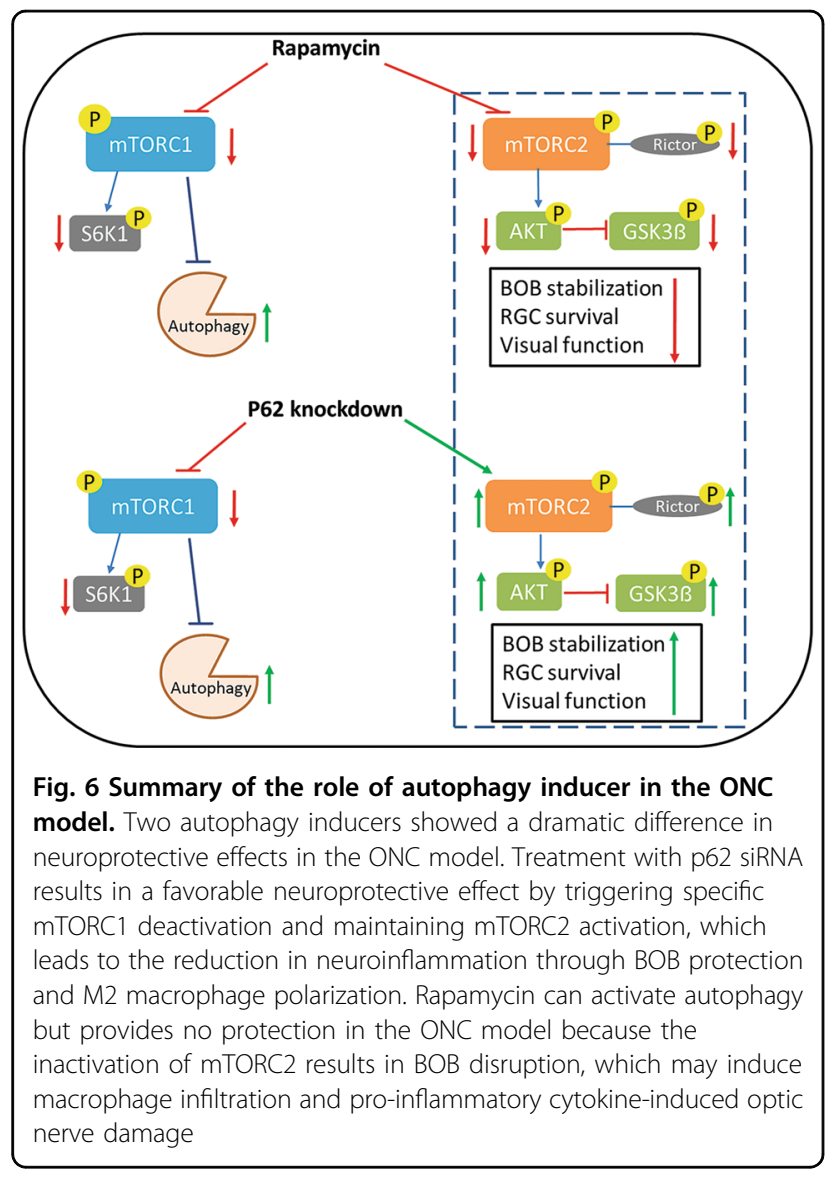

the mTOR pathway. Given the importance of the mTOR pathway, we further narrowed down the pathway involved in the activation of different mTOR complexes. Depending on the type and function of the autophagy inducer, mTOR modulation may vary to different extents. In this study, rapamycin inhibited not only mTORC1 activation but also mTORC2 activation. Inhibition of mTORC2 activation further resulted in Akt and GSK3 $\beta$ inactivation. Akt inactivation led to $\mathrm{BOB}$ disruption, which caused macrophage infiltration into the optic nerve. GSK3 $\beta$ phosphorylation develops as an essential target for the stabilization of the BBB in neuroinflammation ${ }^{43}$. mTORC2 affects biological processes through feedback phosphorylation of Akt, which in turn regulates cell survival mediators and other growth factors, such as GSK3 $\beta$, and components of mitochondrial apoptosis machinery ${ }^{44}$. Several studies have also revealed a strong association of GSK3 $\beta$ phosphorylation with enhanced neuronal growth $^{45-48}$. Consistent with our observations, only P62 knockdown, without inhibition of mTORC2-mediated AKT-GSK3 $\beta$ signaling, provided antiapoptotic effects in the ONC model.

In conclusion, the neuroprotective effects caused by treatment with p62 siRNA involve specific mTORC1 inhibition and mTORC2 activation, which leads to BOB stabilization and M2 macrophage polarization. Treatment with rapamycin can activate autophagy but does not protect RGCs in the ONC model because of the inactivation of mTORC2 inhibition (Fig. 6). This finding suggests an essential role of mTORC2 activation and fills a knowledge gap in the literature on autophagy. Because autophagy is involved in other retinal diseases, such as glaucoma and optic neuropathies, autophagy regulation by using external agents may emerge as a promising clinical application.

\begin{abstract}
Acknowledgements
The authors thank Mr. Yu-Chieh Ho and Ms. I-Ping Tsai for their assistance with animal handling and experiments. We acknowledge Wallace Academic Editing for editing this paper. The Ministry of Science and Technology grant (MOST 103-2314-B-303-007-MY3) and the Taiwan and Buddhist Tzu-Chi General Hospital Grant (TCRD-104-22) supported this study.
\end{abstract}

\section{Authors' contributions}

Conceptualization: R.K.T.; Methodology: Y.T.W., J.R.Z., K.K., R.K.T.; Investigation: R.K.T., J.R.Z.; Writing-original draft: Y.T.W., J.R.Z.; Writing-review and editing: R.K.T., K.K., J.R.Z.; Funding acquisition: R.K.T. All authors have read and approved the final paper.

\section{Author details}

${ }^{1}$ Institute of Eye Research, Hualien Tzu Chi Hospital, Buddhist Tzu Chi Medical Foundation, Hualien, Taiwan. ${ }^{2}$ Department of Ophthalmology, Hualien Tzu Chi Hospital, Buddhist Tzu Chi Medical Foundation, Hualien, Taiwan. ${ }^{3}$ Institute of Medical Sciences, Tzu Chi University, Hualien, Taiwan

\section{Conflict of interest}

The authors declare that they have no conflict of interest.

\section{Publisher's note}

Springer Nature remains neutral with regard to jurisdictional claims in published maps and institutional affiliations.

Supplementary information accompanies this paper at https://doi.org/ 10.1038/s12276-019-0298-z.

Received: 7 November 2018 Revised: 1 May 2019 Accepted: 6 May 2019. Published online: 13 August 2019

\section{References}

1. Tao, W. et al. A novel mouse model of traumatic optic neuropathy using external ultrasound energy to achieve focal, indirect optic nerve injury. Sci. Rep. 7, 11779 (2017).

2. Kumaran, A. M., Sundar, G. \& Chye, L. T. Traumatic optic neuropathy: a review. Craniomaxillofac Trauma Reconstr. 8, 31-41 (2015).

3. Guy, W. M. et al. Traumatic optic neuropathy and second optic nerve injuries. JAMA Ophthalmol. 132, 567-571 (2014).

4. Tsai, R. K., Chang, C. H. \& Wang, H. Z. Neuroprotective effects of recombinant human granulocyte colony-stimulating factor (G-CSF) in neurodegeneration after optic nerve crush in rats. Exp. Eye Res. 87, 242-250 (2008).

5. Tsai, R. K., Chang, C. H., Sheu, M. M. \& Huang, Z. L. Anti-apoptotic effects of human granulocyte colony-stimulating factor (G-CSF) on retinal ganglion cells after optic nerve crush are PI3K/AKT-dependent. Exp. Eye Res. 90, 537-545 (2010).

6. Huang, T. L., Chang, C. H., Lin, K. H., Sheu, M. M. \& Tsai, R. K. Lack of protective effect of local administration of triamcinolone or systemic treatment with methylprednisolone against damages caused by optic nerve crush in rats. Exp. Eye Res. 92, 112-119 (2011). 
7. Huang, S. P. et al. Autocrine protective mechanisms of human granulocyte colony-stimulating factor (G-CSF) on retinal ganglion cells after optic nerve crush. Exp. Eye Res. 143, 132-140 (2016).

8. Oku, H. et al. P7C3 suppresses neuroinflammation and protects retinal ganglion cells of rats from optic nerve crush. Invest Ophthalmol. Vis. Sci. 58, 4877-4888 (2017)

9. Morgan-Warren, P. J. et al. siRNA-mediated knockdown of the mTOR inhibitor RTP801 promotes retinal ganglion cell survival and axon elongation by direct and indirect mechanisms. Invest Ophthalmol. Vis. Sci. 57, 429-443 (2016).

10. Munemasa, Y. \& Kitaoka, Y. Autophagy in axonal degeneration in glaucomatous optic neuropathy. Prog. Retin Eye Res 47, 1-18 (2015).

11. Koch, J. C. \& Lingor, P. The role of autophagy in axonal degeneration of the optic nerve. Exp. Eye Res. 144, 81-89 (2016).

12. Knoferle, J. et al. Mechanisms of acute axonal degeneration in the optic nerve in vivo. Proc. Natl Acad. Sci. USA 107, 6064-6069 (2010).

13. Kim, S. H. et al. Activation of autophagy in retinal ganglion cells. J. Neurosci. Res. 86, 2943-2951 (2008).

14. Rodriguez-Muela, N., Germain, F., Marino, G., Fitze, P. S. \& Boya, P. Autophagy promotes survival of retinal ganglion cells after optic nerve axotomy in mice. Cell Death Differ. 19, 162-169 (2012).

15. Liu, W. J. et al. p62 links the autophagy pathway and the ubiqutin-proteasome system upon ubiquitinated protein degradation. Cell Mol. Biol. Lett. 21, 29 (2016).

16. Huang, S. P., Chien, J. Y. \& Tsai, R. K. Ethambutol induces impaired autophagic flux and apoptosis in the rat retina. Dis. Model Mech. 8, 977-987 (2015).

17. Moscat, J. \& Diaz-Meco, M. T. Feedback on fat: p62-mTORC1-autophagy connections. Cell 147, 724-727 (2011).

18. Produit-Zengaffinen, N., Pournaras, C. J. \& Schorderet, D. F. Autophagy induction does not protect retina against apoptosis in ischemia/reperfusion model. Adv. Exp. Med. Biol. 801, 677-683 (2014).

19. Rodriguez-Muela, N. \& Boya, P. Axonal damage, autophagy and neuronal survival. Autophagy 8, 286-288 (2012).

20. Kitaoka, Y., Kojima, K., Munemasa, Y., Sase, K. \& Takagi, H. Axonal protection by brimonidine with modulation of p62 expression in TNF-induced optic nerve degeneration. Graefes Arch. Clin. Exp. Ophthalmol. 253, 1291-1296 (2015)

21. Sarkar, S., Ravikumar, B., Floto, R. A. \& Rubinsztein, D. C. Rapamycin and mTORindependent autophagy inducers ameliorate toxicity of polyglutamineexpanded huntingtin and related proteinopathies. Cell Death Differ. 16, 46-56 (2009).

22. Schreiber, K. H. et al. Rapamycin-mediated mTORC2 inhibition is determined by the relative expression of FK506-binding proteins. Aging Cell 14, 265-273 (2015).

23. Bjorkoy, G. et al. Monitoring autophagic degradation of p62/SQSTM1. Methods Enzym. 452, 181-197 (2009).

24. Kojima, K. et al. Axonal protection by modulation of p62 expression in TNFinduced optic nerve degeneration. Neurosci. Lett. 581, 37-41 (2014).

25. Duran, A. et al. p62 is a key regulator of nutrient sensing in the mTORC1 pathway. Mol. Cell 44, 134-146 (2011).

26. Lee, J. H. et al. EGCG-mediated autophagy flux has a neuroprotection effect via a class III histone deacetylase in primary neuron cells. Oncotarget $\mathbf{6}$, 9701-9717 (2015)

27. Orihuela, R., McPherson, C. A. \& Harry, G. J. Microglial M1/M2 polarization and metabolic states. Br. J. Pharm. 173, 649-665 (2016).

28. Wen, Y. T., Huang, T. L., Huang, S. P., Chang, C. H. \& Tsai, R. K. Early applications of granulocyte colony-stimulating factor (G-CSF) can stabilize the blood-opticnerve barrier and ameliorate inflammation in a rat model of anterior ischemic optic neuropathy (rAION). Dis. Model Mech. 9, 1193-1202 (2016).
29. Kapupara, K., Wen, Y. T., Tsai, R. K. \& Huang, S. P. Soluble P-selectin promotes retinal ganglion cell survival through activation of Nrf2 signaling after ischemia injury. Cell Death Dis. 8, e3172 (2017).

30. Ito, D. et al. Microglia-specific localisation of a novel calcium binding protein Iba1. Brain Res Mol. Brain Res. 57, 1-9 (1998).

31. Zhang, C., Guo, Y., Miller, N. R. \& Bernstein, S. L. Optic nerve infarction and postischemic inflammation in the rodent model of anterior ischemic optic neuropathy (rAION). Brain Res. 6, 67-75 (2009).

32. Jablonski, K. A. et al. Novel markers to delineate murine M1 and M2 macrophages. PLOS ONE 10, e0145342 (2015).

33. Chi, O. Z., Mellender, S. J., Kiss, G. K., Liu, X. \& Weiss, H. R. Blood -brain barrier disruption was less under isoflurane than pentobarbital anesthesia via a PI3K Akt pathway in early cerebral ischemia. Brain Res. Bull. 131, 1-6 (2017).

34. Wu, F. et al. Acid fibroblast growth factor preserves blood-brain barrier integrity by activating the PI3K-Akt-Rac1 pathway and inhibiting RhoA following traumatic brain injury. Am. J. Transl. Res. 9, 910-925 (2017).

35. Hu, G. W. et al. Exosomes secreted by human-induced pluripotent stem cellderived mesenchymal stem cells attenuate limb ischemia by promoting angiogenesis in mice. Stem Cell Res. Ther. 6, 10 (2015).

36. Chang, C. P., Su, Y. C., Hu, C. W. \& Lei, H. Y. TLR2-dependent selective autophagy regulates NF-kappaB lysosomal degradation in hepatoma-derived M2 macrophage differentiation. Cell Death Differ. 20, 515-523 (2013).

37. Zhang, X. J., Chen, S., Huang, K. X. \& Le, W. D. Why should autophagic flux be assessed? Acta Pharm. Sin. 34, 595-599 (2013).

38. Huang, S., Okamoto, K., Yu, C. \& Sinicrope, F. A. p62/sequestosome-1 upregulation promotes $\mathrm{ABT}$-263-induced caspase-8 aggregation/activation on the autophagosome. J. Biol. Chem. 288, 33654-33666 (2013).

39. Russo, R. et al. Rapamycin and fasting sustain autophagy response activated by ischemia/reperfusion injury and promote retinal ganglion cell survival. Cell Death Dis. 9, 981 (2018).

40. Dumont, F. J. \& Su, Q. Mechanism of action of the immunosuppressant rapamycin. Life Sci. 58, 373-395 (1996).

41. Park, K. K. et al. Promoting axon regeneration in the adult CNS by modulation of the PTEN/mTOR pathway. Science 322, 963-966 (2008).

42. Tassew, N. G. et al. Exosomes mediate mobilization of autocrine Wnt10b to promote axonal regeneration in the injured CNS. Cell Rep. 20, 99-111 (2017).

43. Ramirez, S. H. et al. Inhibition of glycogen synthase kinase 3beta promotes tight junction stability in brain endothelial cells by half-life extension of occludin and claudin-5. PLOS ONE 8, e55972 (2013).

44. Betz, C. et al. Feature Article: mTOR complex 2-Akt signaling at mitochondriaassociated endoplasmic reticulum membranes (MAM) regulates mitochondrial physiology. Proc. Natl Acad. Sci. USA 110, 12526-12534 (2013).

45. Li, R. et al. NGF attenuates high glucose-induced ER Stress, preventing schwann cell apoptosis by activating the PI3K/Akt/GSK3beta and ERK1/2 Pathways. Neurochem Res. 42, 3005-3018 (2017).

46. Liz, M. A. et al. Neuronal deletion of GSK3beta increases microtubule speed in the growth cone and enhances axon regeneration via CRMP-2 and independently of MAP1B and CLASP2. BMC Biol. 12, 1741-7007 (2014).

47. Goold, R. G., Owen, R. \& Gordon-Weeks, P. R. Glycogen synthase kinase 3beta phosphorylation of microtubule-associated protein $1 \mathrm{~B}$ regulates the stability of microtubules in growth cones. J. Cell Sci. 112, 3373-3384 (1999).

48. Fang, W. et al. Role of the Akt/GSK-3beta/CRMP-2 pathway in axon degeneration of dopaminergic neurons resulting from MPP+ toxicity. Brain Res. $\mathbf{3 0}$ 9-19 (2015) 